\title{
Laparoscopy-Assisted versus Open Hepatectomy for Live Liver Donor: Systematic Review and Meta-Analysis
}

\author{
Bin Zhang, ${ }^{1,2}$ Yu Pan, ${ }^{1,2}$ Ke Chen, ${ }^{1,2}$ Hendi Maher, ${ }^{2}$ Ming-Yu Chen, ${ }^{1,2}$ He-Pan Zhu, ${ }^{1,2}$ \\ Yi-Bin Zhu, ${ }^{1,2}$ Yi Dai, ${ }^{1}$ Jiang Chen, ${ }^{1}$ and Xiu-jun Cai ${ }^{1}$ \\ ${ }^{1}$ Department of General Surgery, Sir Run Run Shaw Hospital, School of Medicine, Zhejiang University, \\ 3 East Qingchun Road, Hangzhou, Zhejiang Province 310016, China \\ ${ }^{2}$ School of Medicine, Zhejiang University, 866 Yuhangtang Road, Hangzhou, Zhejiang Province 310058, China \\ Correspondence should be addressed to Xiu-jun Cai; srrsh_cxj@zju.edu.cn
}

Received 22 June 2017; Revised 3 September 2017; Accepted 4 October 2017; Published 7 November 2017

Academic Editor: Kevork M. Peltekian

Copyright (C) 2017 Bin Zhang et al. This is an open access article distributed under the Creative Commons Attribution License, which permits unrestricted use, distribution, and reproduction in any medium, provided the original work is properly cited.

\begin{abstract}
Objective. To assess the feasibility, safety, and potential benefits of laparoscopy-assisted living donor hepatectomy (LADH) in comparison with open living donor hepatectomy $(\mathrm{ODH})$ for liver transplantation. Background. LADH is becoming increasingly common for living donor liver transplant around the world. We aim to determine the efficacy of LADH and compare it with ODH. Methods. A systematic search on PubMed, Embase, Cochrane Library, and Web of Science was conducted in May 2017. Results. Nine studies were suitable for this analysis, involving 979 patients. LADH seemed to be associated with increased operation time $(\mathrm{WMD}=24.85 \mathrm{~min}$; 95\% CI: $-3.01 \sim 52.78, P=0.08$ ), less intraoperative blood loss (WMD $=-59.92 \mathrm{ml}$; 95\% CI: $-94.58 \sim-25.27$, $P=0.0007)$, similar hospital stays (WMD $=-0.47 \mathrm{~d} ; 95 \% \mathrm{CI}:-1.78 \sim 0.83, P=0.47)$, less postoperative complications $(\mathrm{RR}=0.70$, 95\% CI: 0.51 0.94, $P=0.02$ ), less analgesic use (SMD $=-0.22 ; 95 \% \mathrm{CI}:-0.44 \sim-0.11, P=0.04)$, similar transfusion rates $(\mathrm{RR}=$ 0.82; 95\% CI: $0.24 \sim 3.12, P=0.82$ ), and similar graft weights (WMD = 7.31 g; 95\% CI: $-23.45 \sim 38.07, P=0.64$ ). Conclusion. Our results indicate that $\mathrm{LADH}$ is a safe and effective technique and, when compared to ODH.
\end{abstract}

\section{Introduction}

Liver transplantation from living donors is a potential treatment for end-stage liver disease. And due, in part, to the limited number of available livers from deceased patients, living donor liver transplantation (LDLT) has become an established solution. Since the first successful LDLT for a child in 1989 [1], this life-saving procedure has developed rapidly, providing similar or even better outcomes, especially in children, in comparison with cadaver liver grafts [2]. Living donors are typically healthy adults; therefore the donor's safety is paramount.

Over the past two decades, laparoscopic surgery has been widely applied to liver surgery. In 2002, Cherqui et al. [3] reported the first case of laparoscopic living donor left lobectomy and laparoscopic LDLT was increasingly used in some centers. However, owing to technical difficulties, this procedure developed relatively slowly. The first case of laparoscopic-assisted hybrid living donor hepatectomy (LADH) was reported by Koffron et al. [4] in 2006, in which hands were introduced into the abdomen while still maintaining the pneumoperitoneum. In this procedure, a laparoscopic technique is employed for mobilization of liver and hilar dissection; however, the parenchymal transection is performed as an open procedure. As a result, this hybrid procedure achieved the advantage of avoiding a large subcostal incision while retaining the safety and familiarity of an open dissection and resection. In addition, laparoscopic-assisted surgeries offered surgeons an opportunity to accumulate expertise before converting to complete laparoscopic living donor hepatectomies.

Several studies have compared the outcome of laparoscopic-assisted living donor hepatectomy (LADH) with widely used open living donor hepatectomy (ODH). However, no consensus has been reached on this topic; it is still not clear which method is of more benefit to the donor. In 
this setting, we comprehensively collected relevant data and conducted a systematic review with meta-analysis to assess the feasibility, safety, and potential benefits of laparoscopicassisted living donor hepatectomy.

\section{Materials and Methods}

2.1. Systematic Literature Search. This meta-analysis was finished by searching electronic databases of PubMed, Embase, Cochrane Library, and Web of Science and scanning reference lists of articles in May 2017 by Two investigators (B. Zhang and Y. Pan) independently. Strategies included the terms "laparoscopy", "laparoscopic", "minimally invasive", "hybrid", "hand-assisted", "hepatectomy", "liver resection", "hepatic resection", "living donor", and "liver donor". All eligible studies in English were retrieved, and their bibliographies were checked for potential relevant publications.

2.2. Eligibility Criteria. Studies comparing laparoscopyassisted and open living liver donor hepatectomy are included for the systematic review and meta-analysis including prospective or retrospective case series. Studies were excluded if they met any of the following criteria: (1) case reports, letters, reviews, editorials, and studies lacking control groups; (2) studies that did not report the type of surgery or operation data; (3) if dual (or multiple) studies were reported by the same institution and/or authors, only the most recent publication or the highest quality of studies was included. However, articles from the same authors or centers but with different patient cohorts were included.

2.3. Data Extraction and Quality Assessment. Two investigators (M. Y. Chen and H. P. Zhu) independently assessed publications for inclusion and extracted data from eligible studies, including the baseline characteristics, such as first author, publication year, country of region, study type, sample size, and operation outcomes (operation time and intraoperative estimated blood loss) and postoperative outcomes (overall complications and length of hospital stay). The primary outcomes of the study include blood loss, complications, and analgesic use. The secondary outcomes are operation time, transfusion, length of stay, and graft weights. We made attempts to contact corresponding authors for missing data points. Only one author provided requested data for analysis [5].

The quality of the researches was evaluated by The Newcastle-Ottawa Quality Assessment Scale (NOS). The scale ranged from 0 to 9 stars: studies achieving more than or equal to 6 are deemed as good methodologically.

2.4. Statistical Analysis. All analyses were performed with Review Manager Version 5.3 (The Cochrane Collaboration, Oxford, United Kingdom). Risk ratio (RR) with a 95\% confidence interval (CI) was used for the comparison analysis of dichotomous variables. The same continuous parameters were expressed as weighted mean difference (WMD) in the same unit or standard mean difference (SMD) for different unit with 95\% CI. When data in individual studies was presented as median and a range, the means and standard deviations (SDs) were estimated by Hozo et al. [6]. The test of heterogeneity, which indicated between-study variance, was evaluated according to Cochran's test and Higgins-squared statistic [7]. Pooled effects were calculated using a randomeffects model, unless heterogeneity was less than $50 \%$ or $P<$ 0.05. Graphical funnel plots were generated to determine visual inspections for publication bias.

We conduct subgroup analyses in the studies focusing on right lobe hepatectomies $(\mathrm{RH})$ and left lobe hepatectomies (LH).

\section{Results}

3.1. Study Eligibility. A flowchart of the search strategies, containing reasons for excluding studies, is shown in Figure 1. No randomized controlled trials were identified in the records. Nine studies were selected for the final meta-analysis. Five studies $[8,10,12,14,15]$ compared laparoscopy-assisted and open donor right hepatectomy and one study [11] compared left hepatectomy. Two studies $[5,13]$ had data for both right hepatectomy and left hepatectomy comparisons. One study [9] evaluated the safety and feasibility of mixed laparoscopicassisted donor right and left hepatectomies by comparing them with open donor hepatectomies.

A total of 979 patients were included in the analysis with 309 undergoing $\mathrm{LADH}$ (31.5\%) and 670 undergoing $\mathrm{OH}$ (53.2\%). Characteristics of included studies are summarized in Table 1. Four papers were conducted in Japan $[5,10$, 11, 13], two in the United States [8, 9], one in China [15], one in Korea [14], and one in India [12]. Seven of the studies graded morbidity according to the Clavien-Dindo Classification. Four studies reported conversion in 10 cases, including diaphragmatic rupture (1 case), right hepatic vein injury (1 case), and IVC injury (1 case). And the other conversions were not documented in their respective studies. Three studies reported quality of life for donor in the followup period $[11,12,14]$.

The quality of the research included was generally moderate to satisfactory. NOS shows that one out of the nine studies observed had 6 stars, six had 7 stars, and two had 8 stars. Table 2 shows the evaluation of quality according to NOS.

\subsection{Meta-Analysis Results}

\subsubsection{Primary Outcome}

Blood Loss. Intraoperative blood loss during surgery was significantly less for laparoscopy-assisted procedures compared to open ones (WMD $=-59.92 \mathrm{ml}$; 95\% CI: $-94.58 \sim-25.27$, $P=0.0007$ ) (Figure 2). In the subgroup analysis, LADH was a protective effect against blood loss compared with $\mathrm{ODH}$ in RH (WMD = -57.56 ml; 95\% CI: -94.26 -20.87, $P=0.002)$. For the LH group, the results also show that LADH incurred lower blood loss (WMD $=-91.50 \mathrm{ml}$; 95\% CI: $-198.68 \sim 15.67$, $P=0.08)$. Furthermore, the difference was not significant in the mixed group $(\mathrm{WMD}=300 \mathrm{ml} ; 95 \% \mathrm{CI}:-300.93 \sim 900.93$, $P=0.33)$.

Complication. All of the included studies reported complication rate. A reduced postoperative complication rate was 


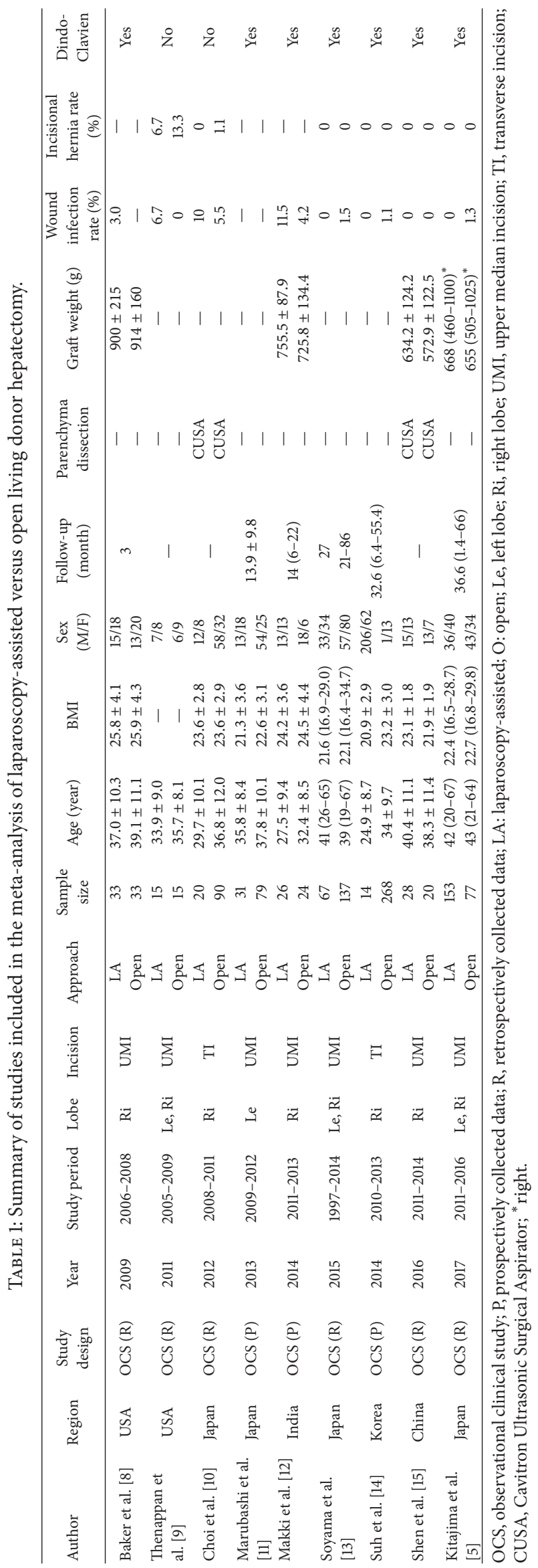


TABLE 2: Quality assessment based on the NOS for observational studies.

\begin{tabular}{|c|c|c|c|c|c|c|c|c|c|c|}
\hline \multirow{2}{*}{ Author } & \multirow{2}{*}{ Matched factors } & \multicolumn{4}{|c|}{ Selection (out of 4) } & \multirow{2}{*}{ Comparability (out of 2) } & \multicolumn{3}{|c|}{ Outcomes (out of 3 ) } & \multirow{2}{*}{ Total (out of 9) } \\
\hline & & (1) & (2) & (3) & (4) & & (5) & (6) & (7) & \\
\hline Baker et al. [8] & abcdef & * & $*$ & $*$ & $*$ & $* *$ & * & & & 7 \\
\hline Thenappan et al. [9] & abcdef & * & * & $*$ & $*$ & $* *$ & * & & & 7 \\
\hline Choi et al. [10] & abcdefghijkl & * & * & $*$ & $*$ & $* *$ & * & & & 7 \\
\hline Marubashi et al. [11] & - & * & * & $*$ & $*$ & $* *$ & * & * & & 8 \\
\hline Makki et al. [12] & abcd & $*$ & * & $*$ & $*$ & * & $*$ & & & 6 \\
\hline Soyama et al. [13] & abcd & * & * & $*$ & * & $* *$ & * & & & 7 \\
\hline Suh et al. [14] & - & * & * & $*$ & * & * & $*$ & $*$ & * & 8 \\
\hline Shen et al. [15] & abcd & * & $*$ & $*$ & * & $* *$ & $*$ & & & 7 \\
\hline Kitajima et al. [5] & - & * & $*$ & $*$ & $*$ & $* *$ & $*$ & & & 7 \\
\hline
\end{tabular}

Factors matched between groups: a: age; b: gender; c: body mass index; d: hepatic artery anomalies; e: portal vein anomalies; f: biliary anomalies; g: ALT; h: AST; i: hemoglobin; j: prothrombin time prothrombin time; k: prothrombin rate; l: international normalized ratio.
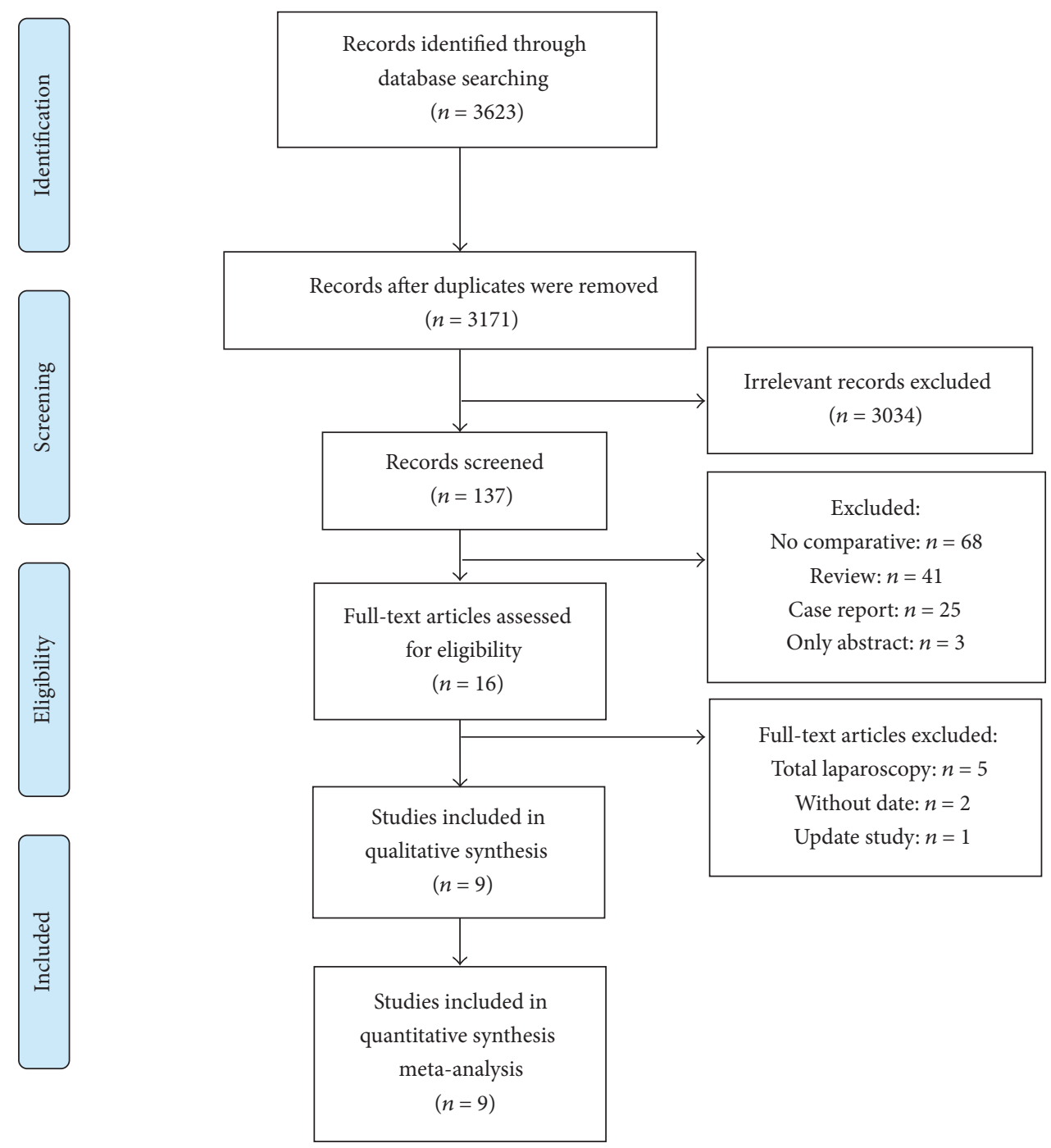

FIgURE 1: Flow diagram of included studies. 


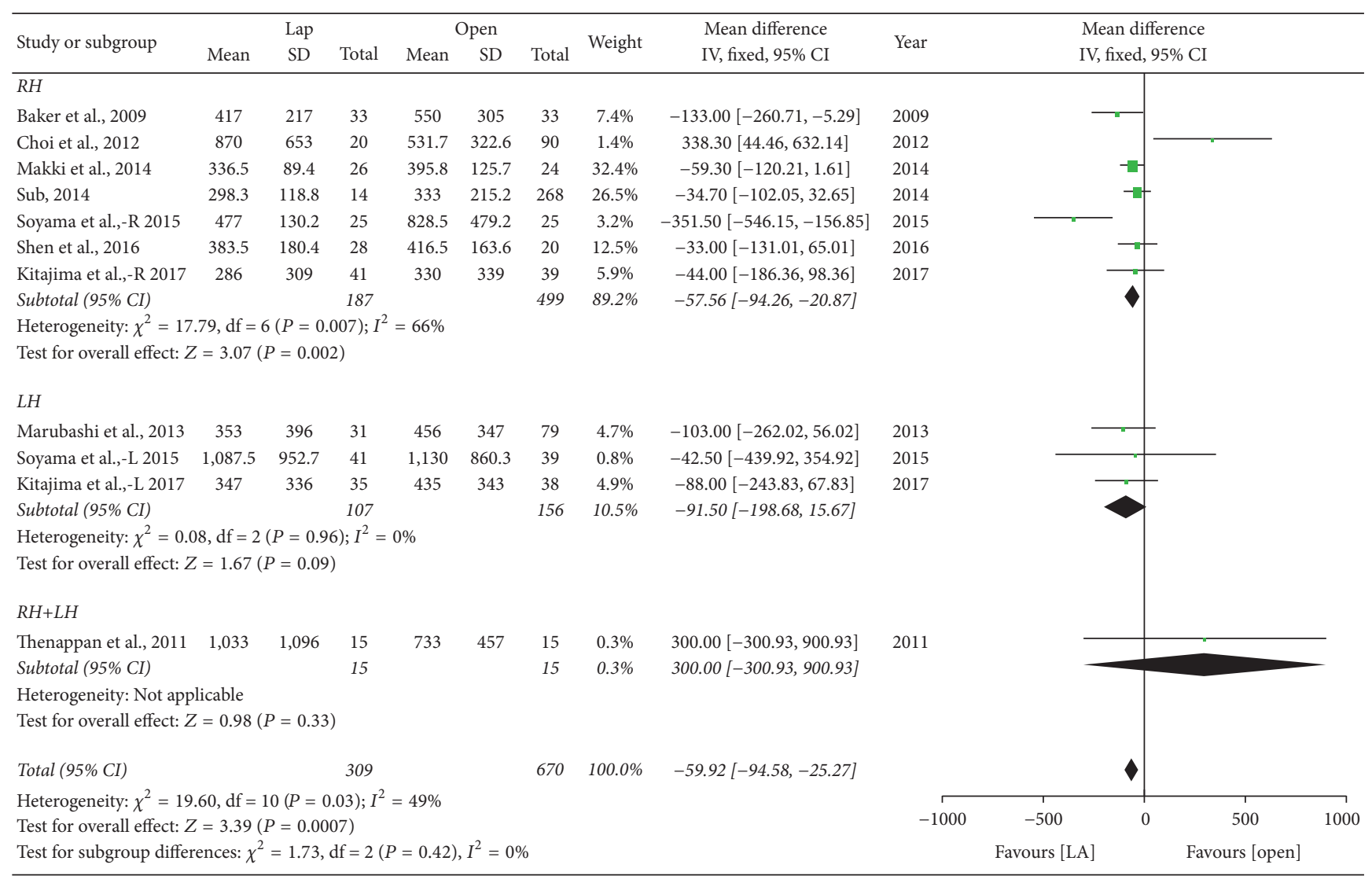

FIGURE 2: Forest plot of subgroup analyses-intraoperative blood loss. Lap: laparoscopy-assisted living donor hepatectomy, Open: open donor hepatectomy, RH: right lobe hepatectomy, LH: left lobe hepatectomy, and $\mathrm{RH}+\mathrm{LH}$ : mixed group.

observed in the LADH group $(\mathrm{RR}=0.70,95 \% \mathrm{CI}: 0.51 \sim$ $0.94, P=0.02$ ) (Figure $3(\mathrm{a})$ ). In the subgroup analysis, $\mathrm{LADH}$ was comparable to $\mathrm{ODH}$ in $\mathrm{RH}$ group $(\mathrm{RR}=0.95$, 95\% CI: $0.63 \sim 1.43, P=0.80)$ and mixed group (RR = 0.59, 95\% CI: 0.29 1.19, $P=0.14$ ). However, complications were significantly decreased in $\mathrm{LADH}$ for $\mathrm{LH}$ procedures $(\mathrm{RR}=0.43,95 \% \mathrm{CI}: 0.23 \sim 0.79, P=0.007)$. There are no differences between the two groups regarding the Clavien grades I to IV and V complications (Figures 3(b), 3(c), and $3(\mathrm{~d})$ ). Postoperative complications included in this study are summarized in Table 3.

Analgesic Use. There are five studies that gave relevant information on analgesic use after surgery and postoperative pain was evaluated by the number of days of analgesic use or the dosage of analgesic. We found that analgesic use was significantly less in the LADH group (SMD $=-0.22 ; 95 \% \mathrm{CI}$ : $-0.44 \sim-0.11, P=0.04$ ) (Figure 4 ).

\subsubsection{Secondary Outcomes}

Operative Time. Nine of the included studies [5, 8-15] reported operation times and mean operation time tended to be longer in $\mathrm{LADH}$ compared to $\mathrm{ODH}(\mathrm{WMD}=24.85 \mathrm{~min}$; 95\% CI: $-3.01 \sim 52.78, P=0.08$ ) (Figure 5). Two of the studies $[5,13]$ provided data for right lobe hepatectomy $(\mathrm{RH})$ and left lobe hepatectomy ( $\mathrm{LH})$, respectively, and we then did a subgroup analysis of $\mathrm{RH}, \mathrm{LH}$, and mixed group. The subgroup analysis shows that there was no significant difference in operation time in $\mathrm{LADH}$ and $\mathrm{ODH}$ groups in $\mathrm{RH}$ (WMD $=23.86 \mathrm{~min}$; 95\% CI: $-13.72 \sim 61.44, P=0.21$ ), $\mathrm{LH}$ (WMD $=20.92 \mathrm{~min} ; 95 \% \mathrm{CI}:-26.85 \sim 68.69, P=0.39)$, and mixed (WMD = $52 \mathrm{~min}$; 95\% CI: $-11.89 \sim 68.894, P=0.11$ ) subgroup.

Transfusion. Five studies reported transfusion information, with similar outcomes in both $\mathrm{LADH}$ and $\mathrm{ODH}(\mathrm{RR}=0.82$; 95\% CI: 0.24 3.12, $P=0.82$ ) (Figure 6).

Length of Hospital Stay. Length of hospital stay was similar between $\mathrm{LADH}$ and $\mathrm{ODH}(\mathrm{WMD}=-0.47 \mathrm{~d}$; $95 \% \mathrm{CI}:-1.78 \sim$ $0.83, P=0.47$ ) (Figure 7 ). For the subgroup analysis, there were no significant difference between $\mathrm{LADH}$ and $\mathrm{ODH}$ in the $\mathrm{RH}$ group $(\mathrm{WMD}=-0.84 \mathrm{~d}$; $95 \% \mathrm{CI}:-2.58 \sim 0.91, P=$ 0.35), LH (WMD = $1.00 \mathrm{~d}$; 95\% CI: $-1.64 \sim 3.64, P=0.46)$, or the mixed group (WMD $=-0.40 \mathrm{~d} ; 95 \% \mathrm{CI}:-2.52 \sim 1.72$, $P=0.71)$.

3.2.3. Graft Weight. A total of 4 studies reported graft weight, showing no difference between the two groups (WMD = 7.31 g; 95\% CI: $-23.45 \sim 38.07, P=0.64$ ) (Figure 8).

3.2.4. Publication Bias. A funnel plot for studies reporting RRs of postoperative overall complications was used to detect publication bias. The plots standing for the studies distributed 


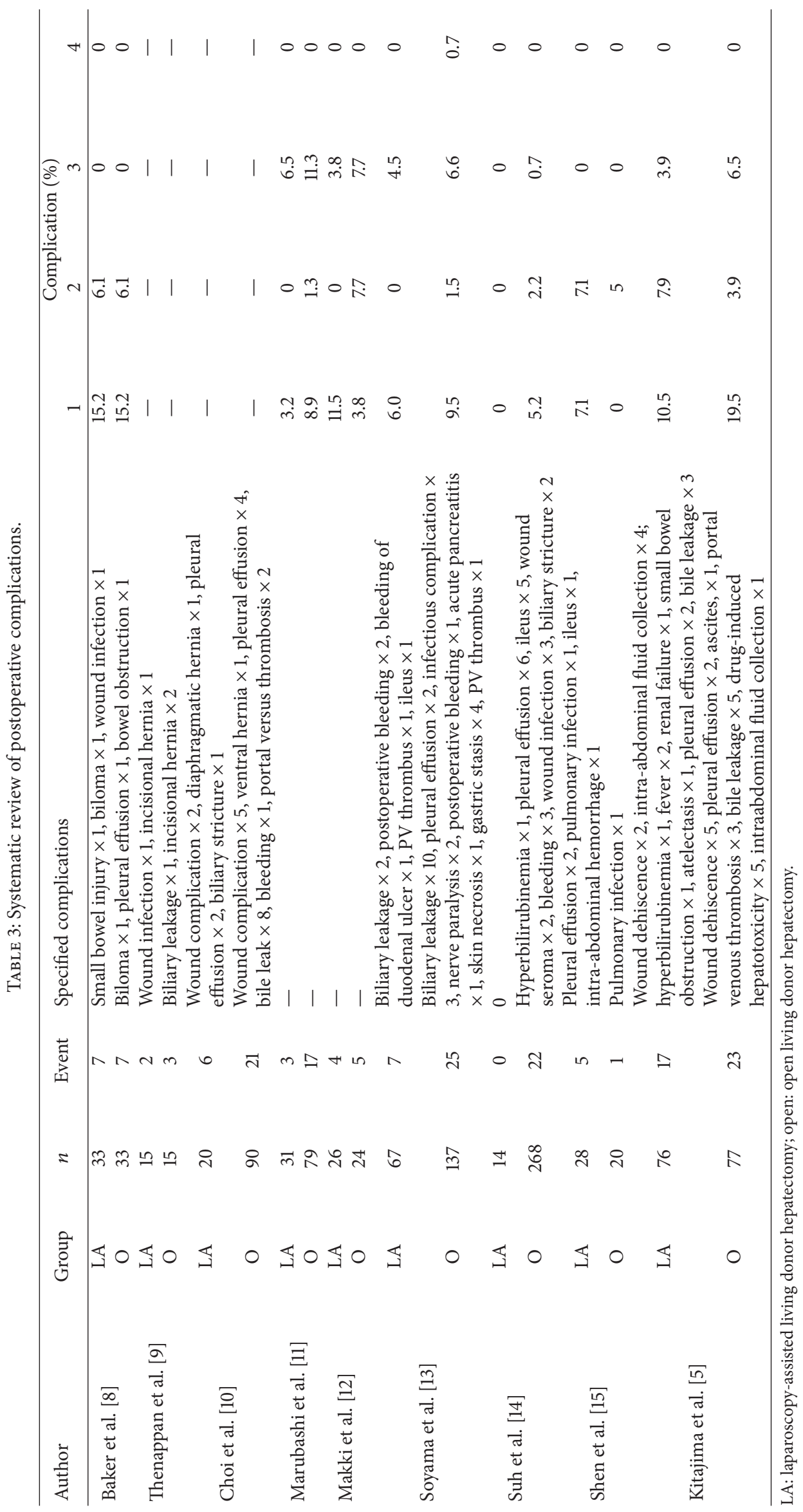




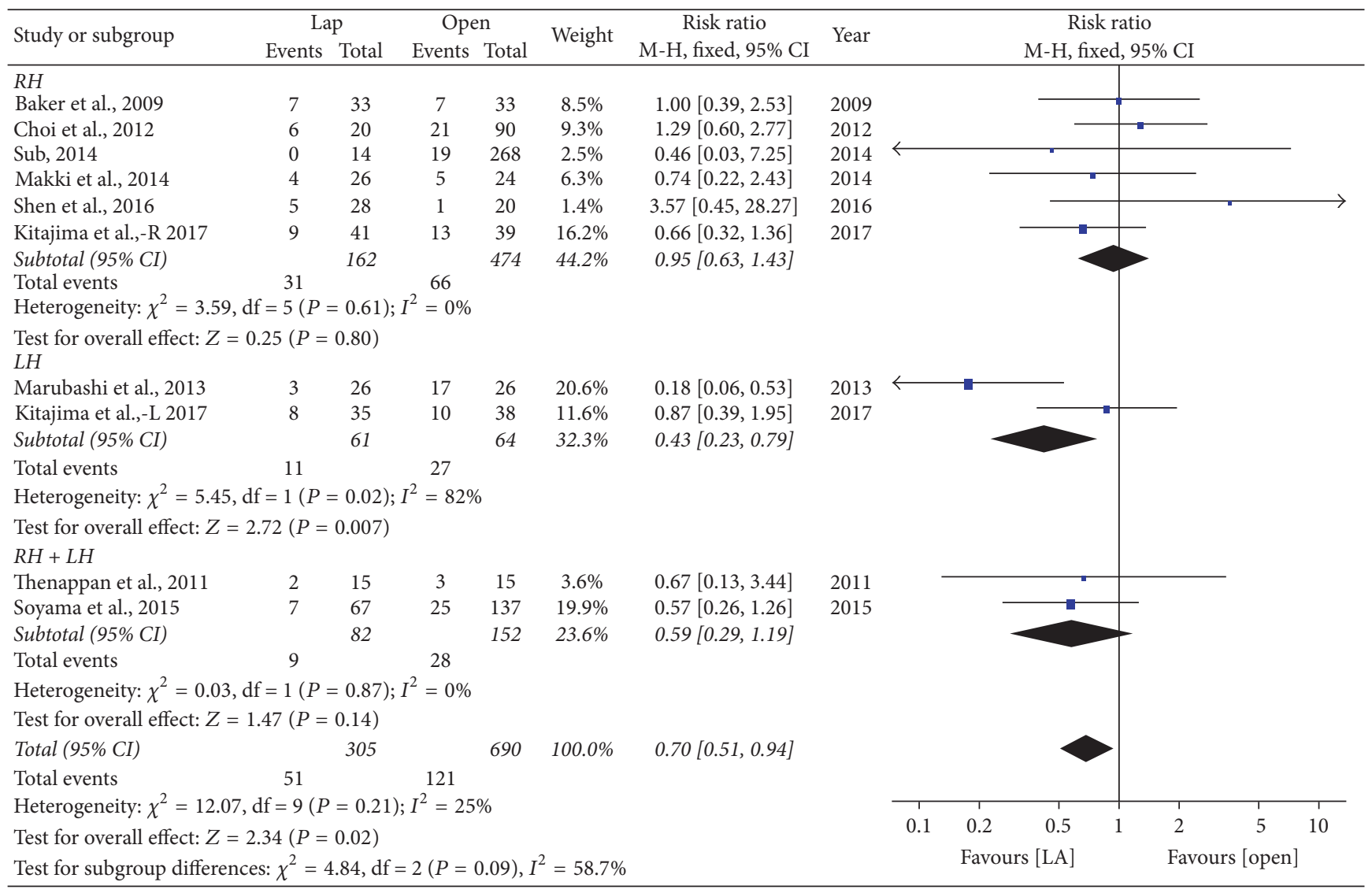

(a)

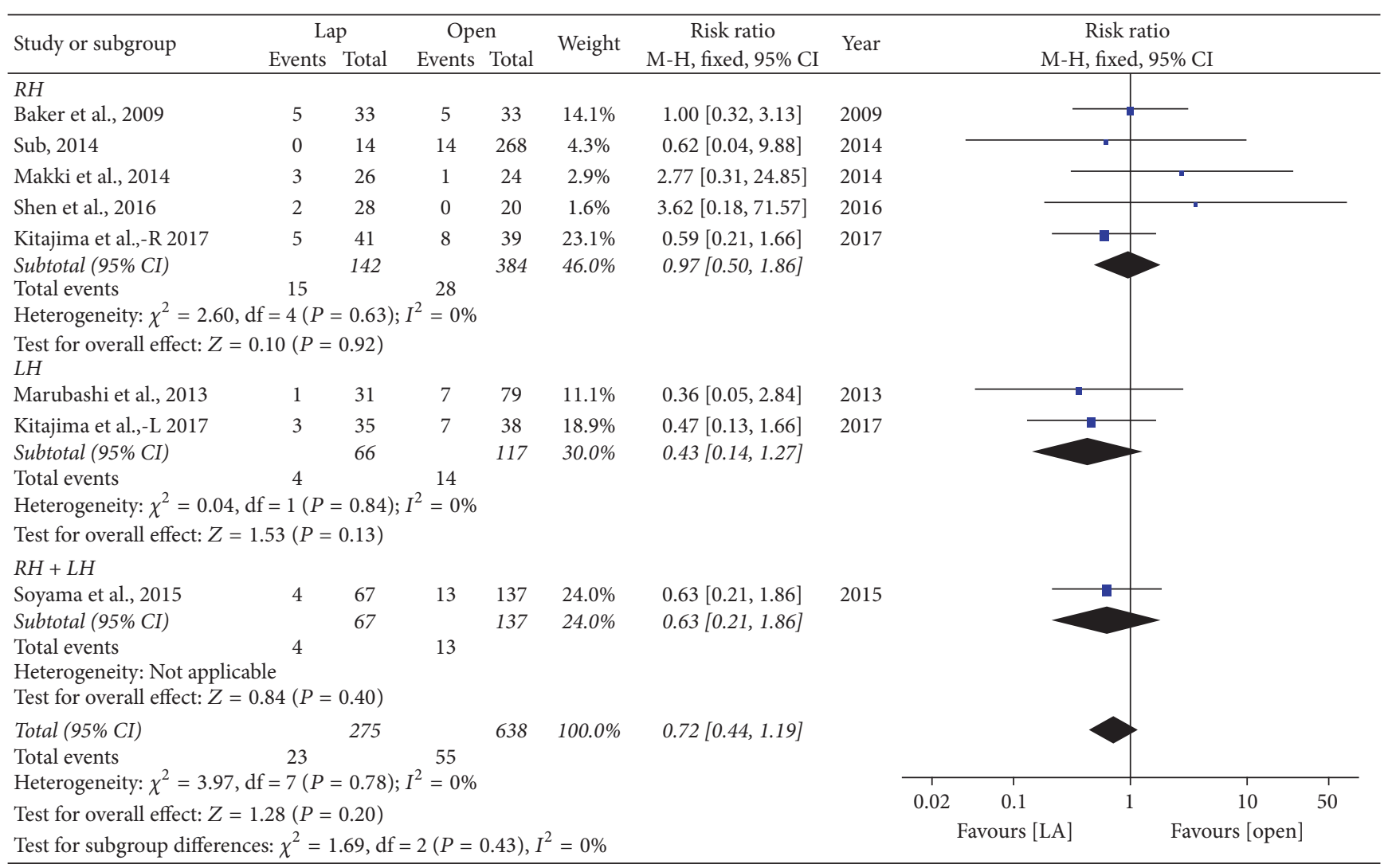

(b)

FIgure 3: Continued. 


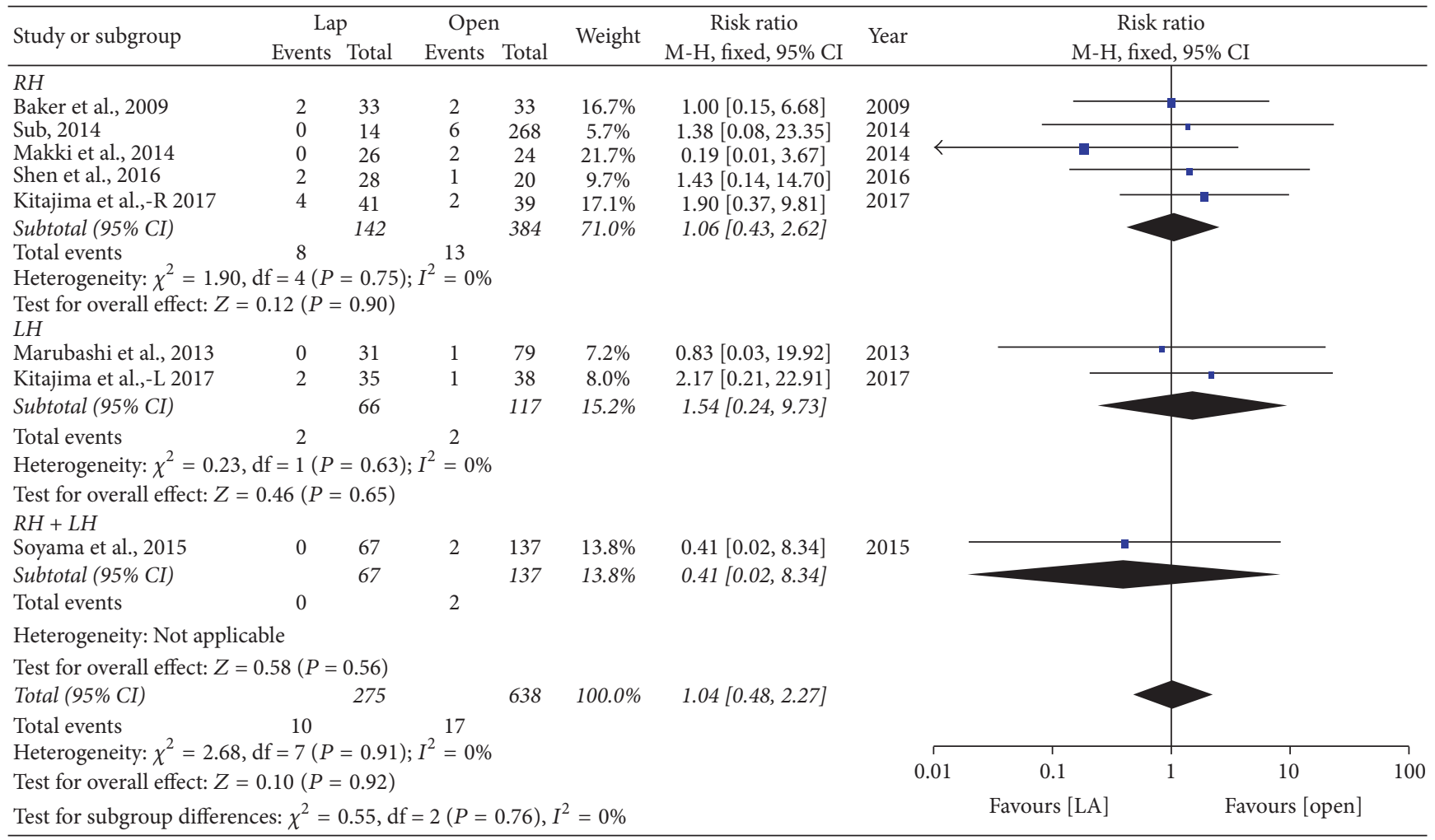

(c)

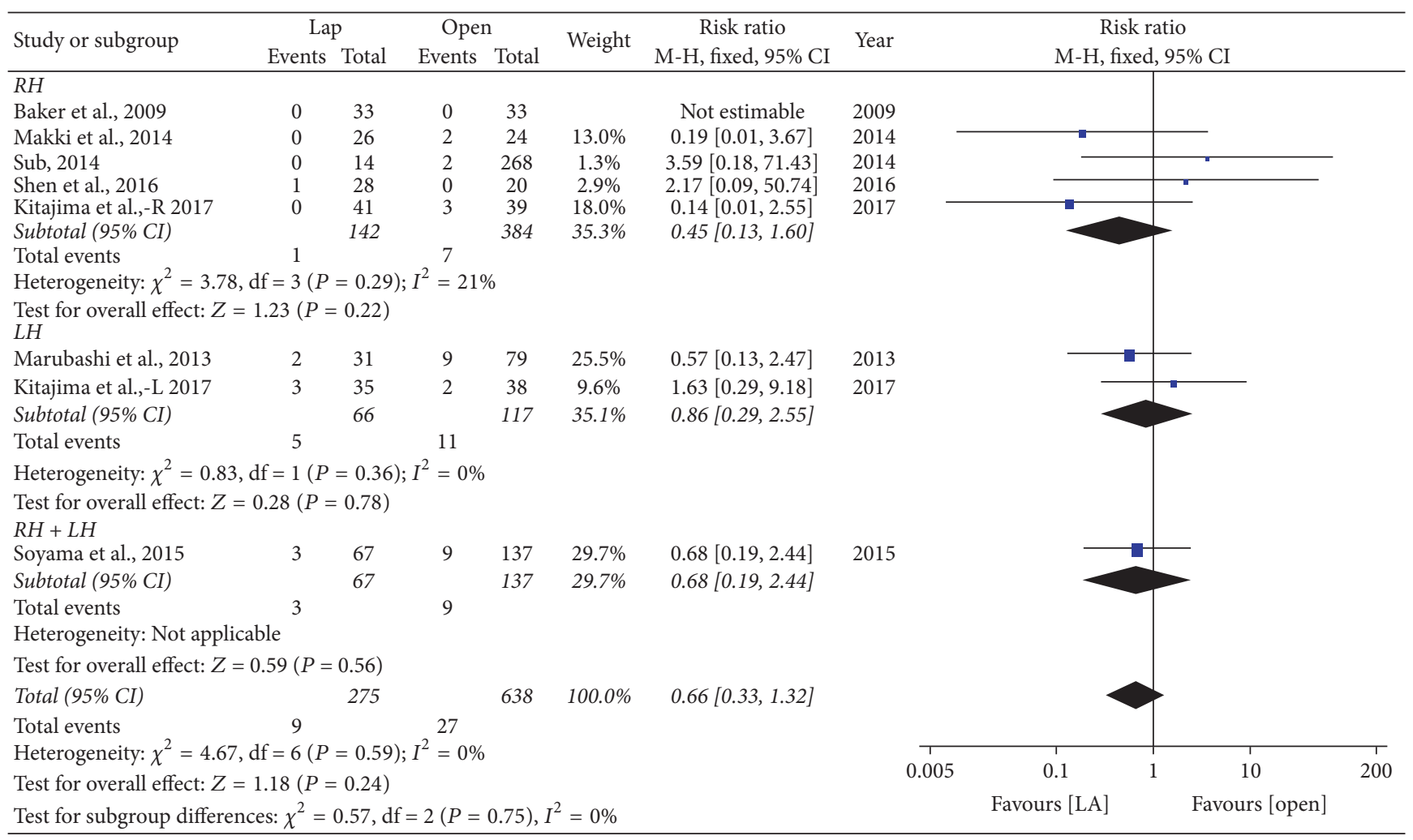

(d)

FIGURE 3: Forest plot of subgroup analyses. (a) Overall postoperative complications. (b) Clavien grade I complication. (c) Clavien grade II complication. (d) Clavien grade III complication. Lap: laparoscopy-assisted living donor hepatectomy, Open: open donor hepatectomy, RH: right lobe hepatectomy, LH: left lobe hepatectomy, and $\mathrm{RH}+\mathrm{LH}$ : mixed group. 


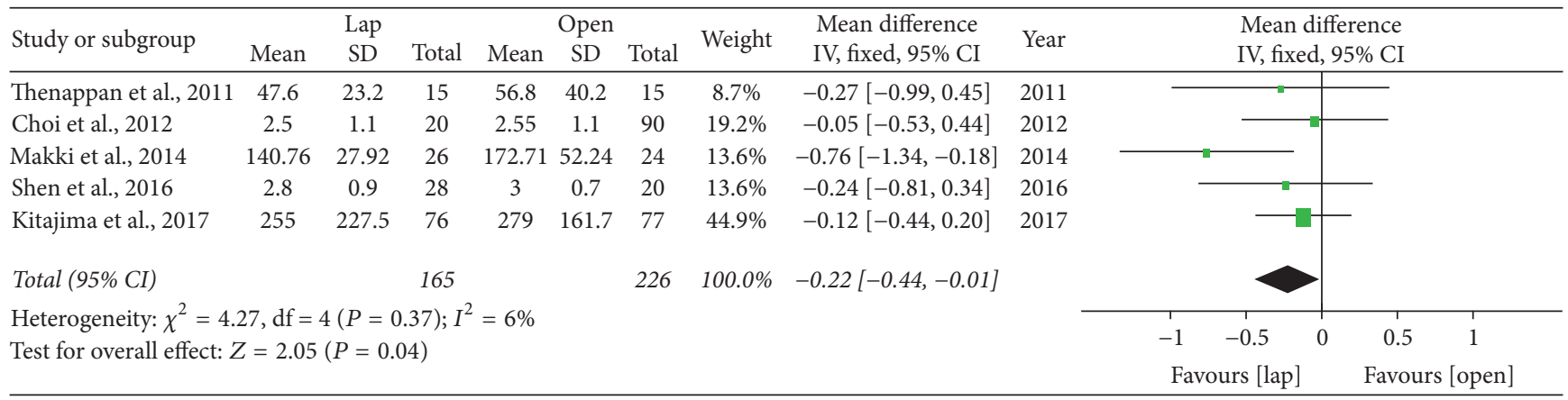

FIGURE 4: Forest plot of meta analyses-analgesic use.

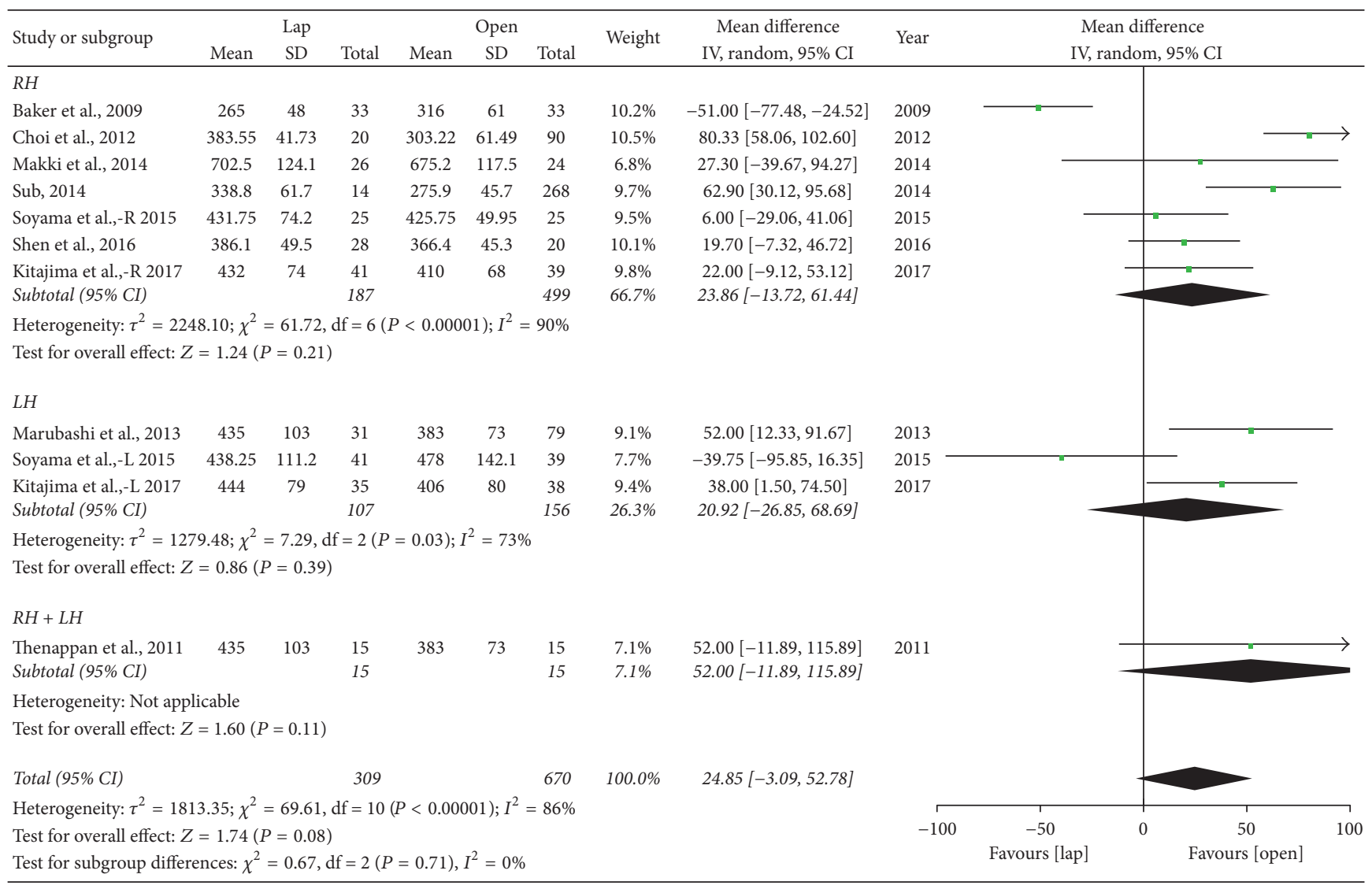

Figure 5: Forest plot of subgroup analyses-operation time. Lap: laparoscopy-assisted living donor hepatectomy, Open: open donor hepatectomy, RH: right lobe hepatectomy, LH: left lobe hepatectomy, and $\mathrm{RH}+\mathrm{LH}$ : mixed group.

symmetrically. This result suggested that the publication bias was acceptable (Figure 9).

\section{Discussion}

Minimally invasive donor surgery was developed to reduce the morbidity and decrease the impact on the donor, minimizing tissue trauma, and improving postoperative pain and cosmesis for patients. LADH with manual hand manipulation in the abdominal cavity, giving the surgeon enhanced tactile feedback of the liver, allowed for more precise mobilization and dissection of the targeted lobe. This technique is combined with smaller incision while preserving the maneuverability and safety of an open liver resection. LADH apparently leads to less wound-related morbidity and the best cosmetic result [16]. In a recent review, Xu et al. [17] examined laparoscopic versus open liver resection for liver transplantation, showing less blood loss, shortened hospital stay, and longer operation time. However, this review did not attempt to clarify the different types of laparoscopic 


\begin{tabular}{lcccccccc|c}
\hline Study or subgroup & \multicolumn{2}{c}{ Lap } & \multicolumn{2}{c}{ Open } & Weight & $\begin{array}{c}\text { Risk ratio } \\
\text { M-H, fixed, 95\% CI }\end{array}$ & Year & & \multicolumn{2}{c}{ Risk ratio } \\
Events, fixed, 95\% CI
\end{tabular}

FIGURE 6: Forest plot of meta analyses—transfusion. Lap: laparoscopy-assisted living donor hepatectomy, Open: open donor hepatectomy.

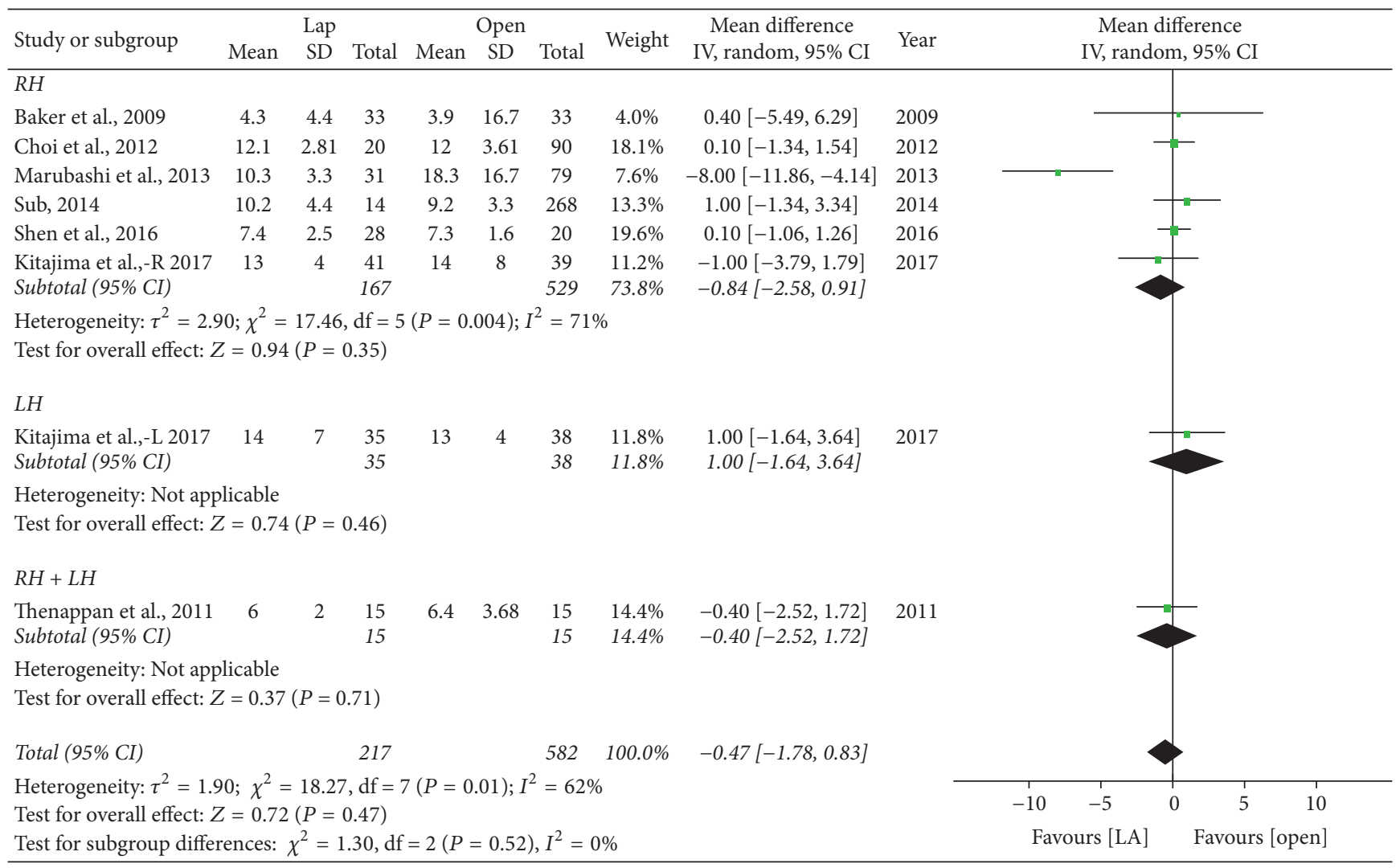

FIGURE 7: Forest plot of subgroup analyses-length of hospital stay. Lap: laparoscopy-assisted living donor hepatectomy, Open: open donor hepatectomy, RH: right lobe hepatectomy, LH: left lobe hepatectomy, and $\mathrm{RH}+\mathrm{LH}$ : mixed group.

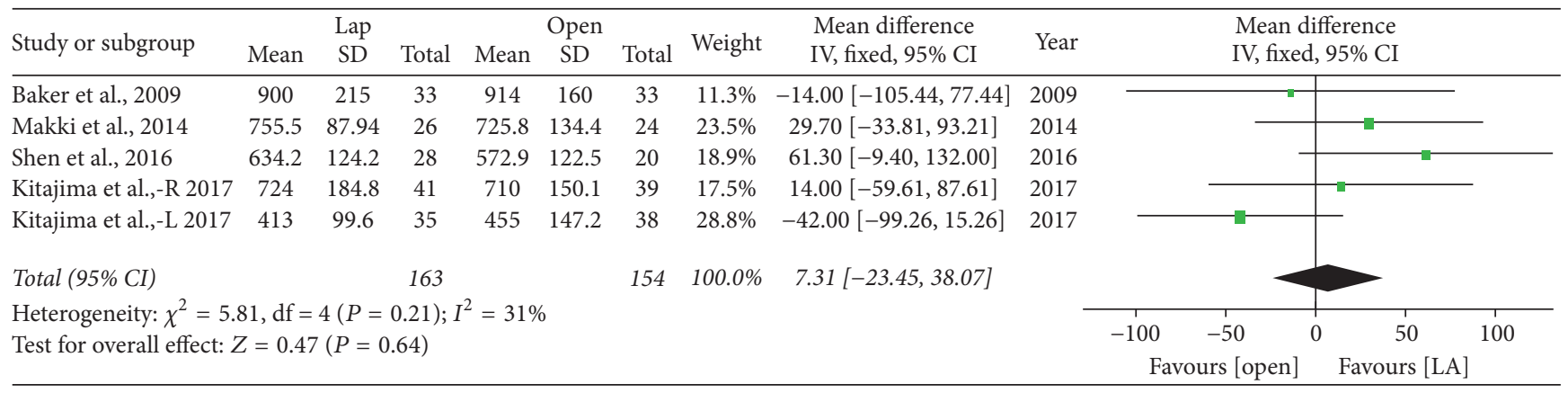




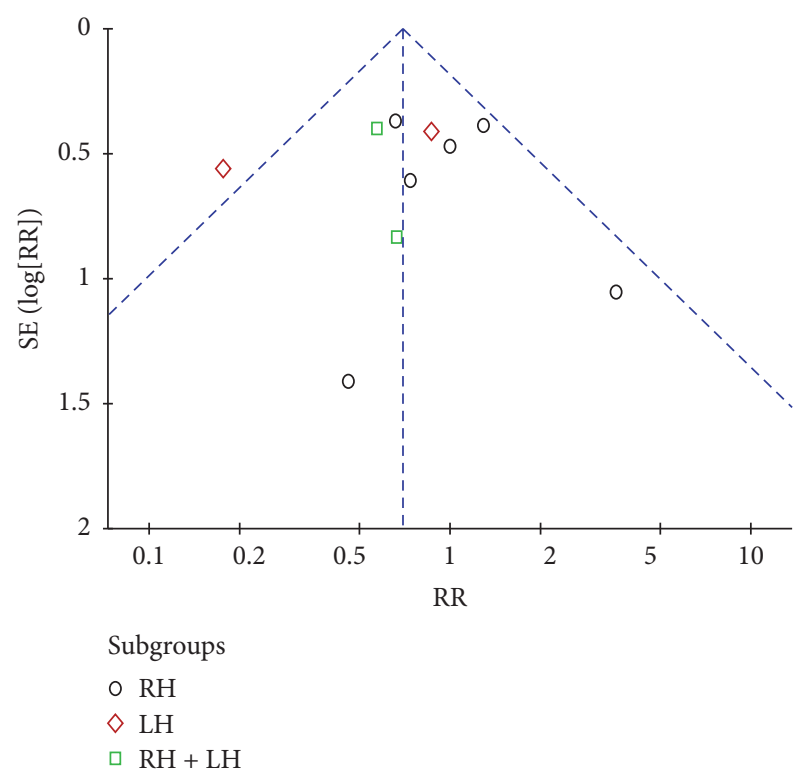

FIGURE 9: Funnel plot of overall postoperative complications. RH: right lobe hepatectomy, LH: left lobe hepatectomy, and $\mathrm{RH}+\mathrm{LH}$ : mixed group.

surgery. In our meta-analysis, we only included the studies of laparoscopy-assisted (hybrid) surgery. Our further subgroup analysis was done to learn how LADH affects surgery in different areas of the liver.

Our result confirms that blood loss was significantly less in the LADH group than in the ODH group. This is consistent with published results for laparoscopic hepatectomies, even when laparoscopy is only used for the hepatic mobilization [18]. In the subgroup analysis of single types of hepatectomy to minimize the bias, there was no difference between the types of donor hepatectomy. LADH is a potential technique to decrease blood loss, confirmed by the colorectal surgery [19] and prior analysis [20]. Hand-assisted surgery has been promoted by its advocates in decreased complication rate in the colorectal surgery [19]. Our analysis of LADH demonstrated favourable overall complication rates compared to ODH, similar to the previous analysis [20]. In the subgroup analysis, LH shows a significantly lower rate of complications in the $\mathrm{LADH}$ group, which accounts for the lower complication rate in the total group. However, the case volume is small in the left hepatectomy subgroup. In theory, it is easier to mobilize the right lobe from the diaphragm by laparoscopic technique and inferior vena cava with the help of manual manipulation. Adequate mobilization, improved visualization, and better manipulation contribute to the enhanced safety of the operation. Living donor mortality in ODH was reported as $0.2 \%(23 / 1153)$, mostly related to surgical procedure [21]. There was no mortality to be reported in the studies both in laparoscopy-assisted and open group for donor. In other words, LADH shows a better tendency toward in the outcome of morbidity to ODH.

Smaller and midline incisions in the supraumbilical area resulted in reduced disruption of abdominal muscles, deceased scar discomfort, and less postoperative analgesic use in our analysis, raising the possibility of better cosmetic results and, possibly, faster return to work and normal physical activities. However, it tended to have an increased operative time associated with hand-assisted surgery, though it did not reach statistical significance. The result could be explained by the application of laparoscopic instruments for the meticulous mobilization in the liver surgery. Furthermore, the transfusion rate was comparable between LADH and ODH in this analysis. Additionally, LOS demonstrated no inferiority for LADH. Interestingly, the prior meta-analysis of laparoscopic versus open hepatectomy for live liver donor has shown the significantly shorter hospital stay in the LADH group $[17,20]$. This may be ascribed to the methods of surgery and postoperation protocols and insurance policy. Regarding hospital cost, it was higher in the LADH. From published data, the overall cost of laparoscopic liver resection was lower than open liver resection [22].

After comparing laparoscopic-assisted operation and open operation, there was a high heterogeneity in the analysis, even in the subgroup analyses by type of surgery. These may result from differences in study designs, number of participants, donors' baseline characteristics, surgical techniques, and surgical types. In addition, some of the data estimated the mean and SD from median and range, which may result in inaccuracy. No random trials were included and most of the studies were cohort studies or case-control studies. Because of high-risk in the donor hepatectomy, a relative surgical abstention may present in the enrolled patients and their families. Based on these limitations, larger prospective studies and randomized trials are needed.

\section{Conclusion}

According to our data, laparoscopy-assisted living donor hepatectomy (LADH) is equally safe and effective technique. There was no increased risk of morbidity compared to $\mathrm{ODH}$ patients in our examined groups. Benefits of laparoscopy-assisted donor hepatectomy compared to open surgery have demonstrated improved short-term outcomes, especially lower intraoperative blood loss and complications. We conclude that LADH is an appropriate minimally invasive procedure for living donor hepatectomies, which needs to be selected by patients' and surgery' preferences.

\section{Abbreviations}

LADH: Laparoscopy-assisted living donor hepatectomy

ODH: Open donor hepatectomy

LLR: Laparoscopic liver resection

WMD: Weighted mean difference

SMD: Standard mean difference

RR: $\quad$ Risk ratio

SD: $\quad$ Standard deviation

NOS: Newcastle-Ottawa Quality Assessment Scale

RH: Right lobe hepatectomy

LH: Left lobe hepatectomy

ALT: Alanine aminotransferase

AST: Aspartate aminotransferase

TB: Total bilirubin

LFT: Liver function test. 


\section{Conflicts of Interest}

The authors declare that they have no conflicts of interest.

\section{Authors' Contributions}

Bin Zhang and Yu Pan wrote the paper and performed the research. Xiu-jun Cai and Ke Chen designed the study. MingYu Chen and He-Pan Zhu collected the data. Hendi Maher and Yi-Bin Zhu performed the literature search and retrieved the data. Xiu-jun Cai, Jiang Chen, and Yi Dai analyzed the data and revised the manuscript.

\section{Acknowledgments}

This study was supported by Research Project of Zhejiang Provincial Education Department (no. N20140173).

\section{References}

[1] S. Raia, J. R. Nery, and S. Mies, "Liver transplantation from live donors," The Lancet, vol. 334, no. 8661, p. 497, 1989.

[2] D. G. Maluf, R. T. Stravitz, A. H. Cotterell et al., "Adult living donor versus deceased donor liver transplantation: A 6-year single center experience," American Journal of Transplantation, vol. 5, no. 1, pp. 149-156, 2005.

[3] D. Cherqui, O. Soubrane, E. Husson et al., "Laparoscopic living donor hepatectomy for liver transplantation in children," The Lancet, vol. 359, no. 9304, pp. 392-396, 2002.

[4] A. J. Koffron, R. Kung, T. Baker, J. Fryer, L. Clark, and M. Abecassis, "Laparoscopic-assisted right lobe donor hepatectomy," American Journal of Transplantation, vol. 6, no. 10, pp. 2522-2525, 2006.

[5] T. Kitajima, T. Kaido, T. Iida et al., "Short-term outcomes of laparoscopy-assisted hybrid living donor hepatectomy: a comparison with the conventional open procedure," Surgical Endoscopy, pp. 1-10, 2017.

[6] S. P. Hozo, B. Djulbegovic, and I. Hozo, "Estimating the mean and variance from the median, range, and the size of a sample," BMC Medical Research Methodology, vol. 5, article 13, 2005.

[7] J. P. T. Higgins, S. G. Thompson, J. J. Deeks, and D. G. Altman, "Measuring inconsistency in meta-analyses," British Medical Journal, vol. 327, no. 7414, pp. 557-560, 2003.

[8] T. B. Baker, C. L. Jay, D. P. Ladner et al., "Laparoscopy-assisted and open living donor right hepatectomy: A comparative study of outcomes," Surgery, vol. 146, no. 4, pp. 817-825, 2009.

[9] A. Thenappan, R. C. Jha, T. Fishbein et al., "Liver allograft outcomes after laparoscopic-assisted and minimal access live donor hepatectomy for transplantation," The American Journal of Surgery, vol. 201, no. 4, pp. 450-455, 2011.

[10] H. J. Choi, Y. K. You, G. H. Na, T. H. Hong, G. S. Shetty, and D. G. Kim, "Single-port laparoscopy-assisted donor right hepatectomy in living donor liver transplantation: Sensible approach or unnecessary hindrance?" Transplantation Proceedings, vol. 44, no. 2, pp. 347-352, 2012.

[11] S. Marubashi, H. Wada, K. Kawamoto et al., "Laparoscopyassisted hybrid left-side donor hepatectomy," World Journal of Surgery, vol. 37, no. 9, pp. 2202-2210, 2013.

[12] K. Makki, V. K. Chorasiya, G. Sood, P. K. Srivastava, P. Dargan, and V. Vij, "Laparoscopy-assisted hepatectomy versus conventional (open) hepatectomy for living donors: When you know better, you do better," Liver Transplantation, vol. 20, no. 10, pp. 1229-1236, 2014.

[13] A. Soyama, M. Takatsuki, M. Hidaka et al., "Hybrid procedure in living donor liver transplantation," Transplantation Proceedings, vol. 47, no. 3, pp. 679-682, 2015.

[14] S.-W. Suh, K.-W. Lee, J.-M. Lee, Y. Choi, N.-J. Yi, and K.-S. Suh, "Clinical outcomes of and patient satisfaction with different incision methods for donor hepatectomy in living donor liver," Liver Transplantation, vol. 21, no. 1, pp. 72-78, 2015.

[15] S. Shen, W. Zhang, L. Jiang, L. Yan, and J. Yang, "Comparison of Upper Midline Incision With and Without Laparoscopic Assistance for Living-Donor Right Hepatectomy," Transplantation Proceedings, vol. 48, no. 8, pp. 2726-2731, 2016.

[16] A. Jain, P. Nemitz, R. Sharma et al., "Incidence of abdominal wall numbness post-liver transplantation and its complications," Liver Transplantation, vol. 15, no. 11, pp. 1488-1492, 2009.

[17] J. Xu, C. Hu, H.-L. Cao et al., "Meta-analysis of laparoscopic versus open hepatectomy for live liver donors," PLOS ONE, vol. 11, no. 10, Article ID e0165319, 2016.

[18] H.-S. Han, A. Shehta, S. Ahn, Y.-S. Yoon, J. Y. Cho, and Y. Choi, "Laparoscopic versus open liver resection for hepatocellular carcinoma: Case-matched study with propensity score matching," Journal of Hepatology, vol. 63, no. 3, article no. 5641, pp. 643-650, 2015.

[19] H. Moloo, F. Haggar, D. Coyle et al., "Hand assisted laparoscopic surgery versus conventional laparoscopy for colorectal surgery," Cochrane Database of Systematic Reviews, no. 10, Article ID CD006585, 2010.

[20] G. Berardi, F. Tomassini, and R. I. Troisi, "Comparison between minimally invasive and open living donor hepatectomy: A systematic review and meta-analysis," Liver Transplantation, vol. 21, no. 6, pp. 738-752, 2015.

[21] Y. L. Cheah, M. A. Simpson, J. J. Pomposelli, and E. A. Pomfret, "Incidence of death and potentially life-threatening near-miss events in living donor hepatic lobectomy: a world-wide survey," Liver Transplantation, vol. 19, no. 5, pp. 499-506, 2013.

[22] S. P. Cleary, H.-S. Han, M. Yamamoto, G. Wakabayashi, and H. J. Asbun, "The comparative costs of laparoscopic and open liver resection: a report for the 2 nd International Consensus Conference on Laparoscopic Liver Resection," Surgical Endoscopy, vol. 30, no. 11, pp. 4691-4696, 2016. 


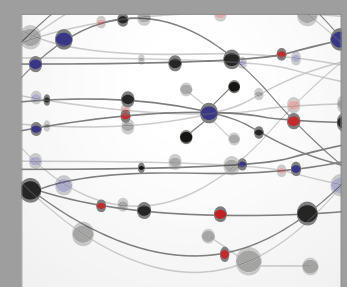

The Scientific World Journal
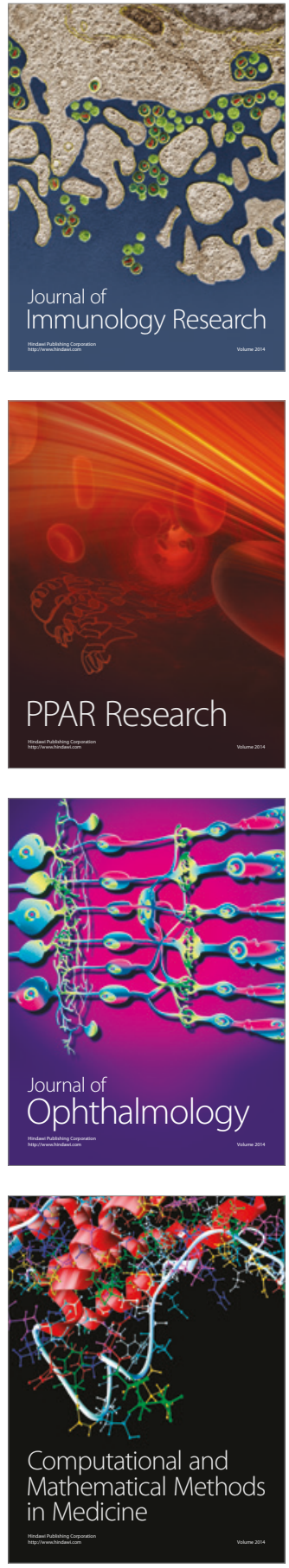

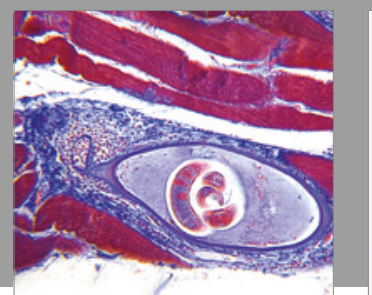

Gastroenterology Research and Practice
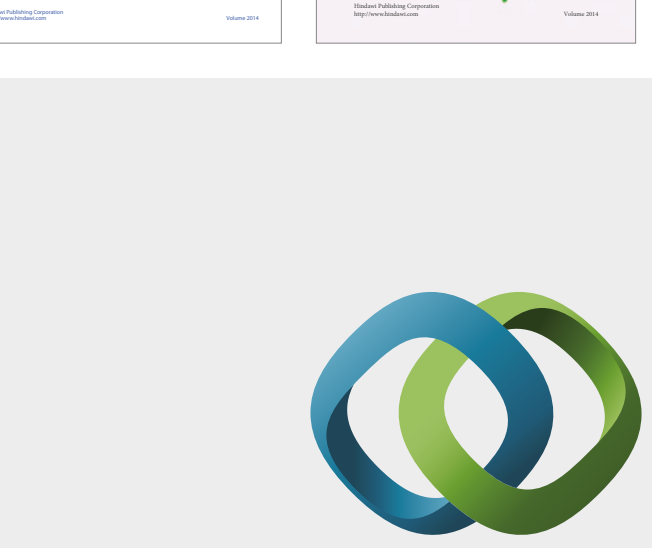

\section{Hindawi}

Submit your manuscripts at

https://www.hindawi.com
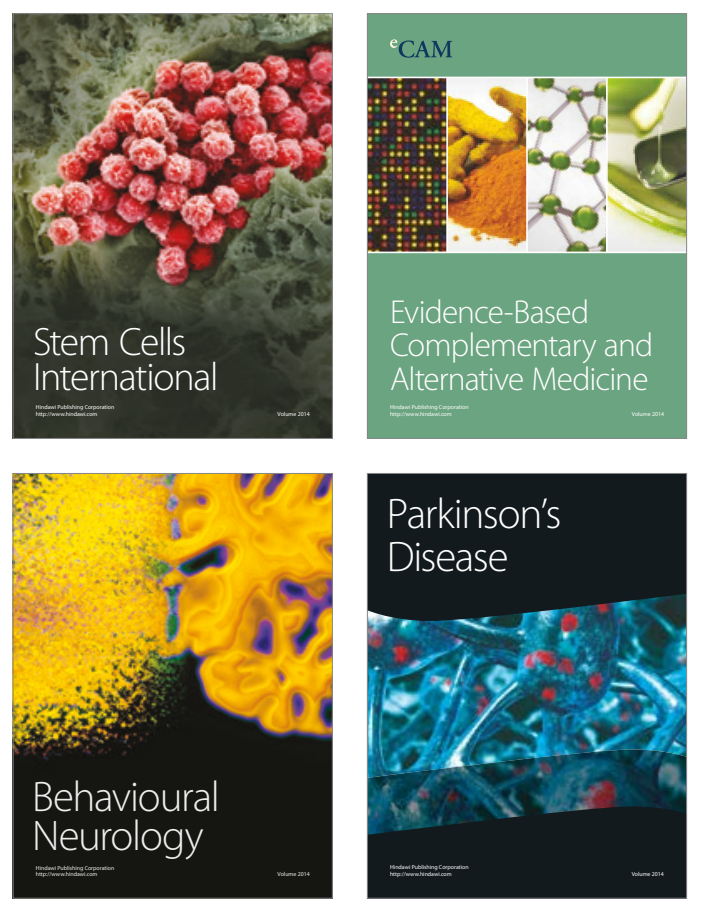
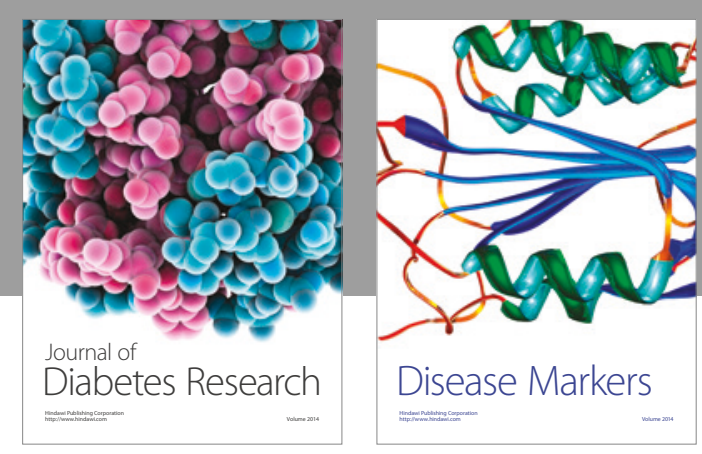

Disease Markers
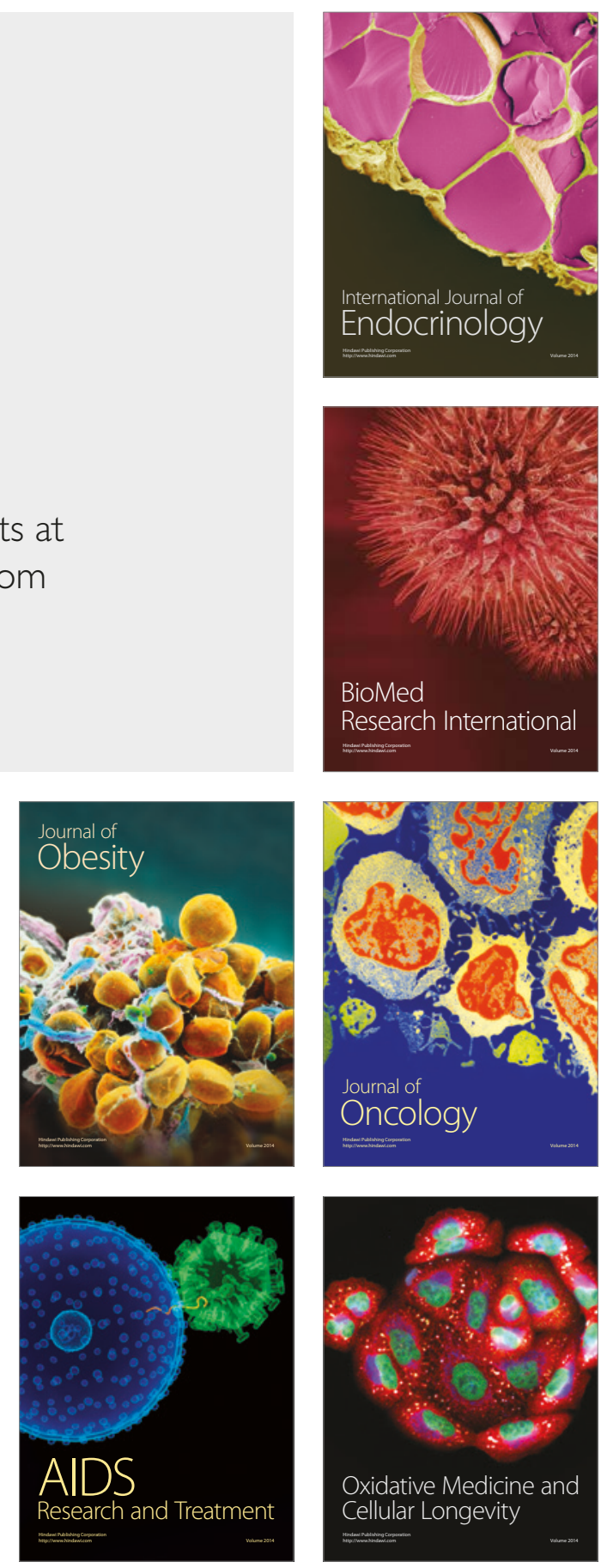